to contain calcium. All these solutions contain hypochlorites and are unstable to a varying extent.

\section{HYDROLYSIS OF D.E.S.}

Although sodium bicarbonate solution was probably suggested originally as an eye-wash in the hope of producing hydrolysis, and irrigation by saturated boric acid followed by sodium bicarbonate ointment (Kinttof, 1939) is still the German first-aid treatment, it is doubtful if hydrolysis of mustard gas in the conjunctival sac plays much part in reducing the severity of the lesion, for the following reasons:

(a) At $10^{\circ}$ C. mustard gas has a solubility of only 0.07 per cent. in water. At body temperature 25 per cent. of the mustard gas in such a solution is hydrolysed in three minutes, but this reaction is not accelerated by alkalis (Peters and Walker, 1923).

(b) The tears, except in the earliest stages of the burn, have no vesicant effect on the conjunctiva of rabbits (Warthin). (Bonnefon dissents from this, stating that tears are vesicant for two to five days.)

(c) If mustard gas is applied to skin covered by a paste of sodium bicarbonate and water the resultant burn is worse than if it is applied directly to the skin (Warthin).

Consideration of these facts suggests that if sodium bicarbonate solution has any effect on eye burns it is simply by virtue of the irrigant effect of the aqueous vehicle. Saturated boric acid acts in the same way, but has the disadvantage that it dissolves slowly and that solutions must be prepared in quantity in advance.

\section{Hypertonic Withdrawal}

Bonnefon has advocated the use of hypertonic solutions on the grounds that a freer flow of lymph is produced in the already oedematous conjunctiva, and that D.E.S. is withdrawn through the conjunctiva by the osmotic effect of the solution-as shown by the fact that the fluid in the eye-bath used for application becomes caustic after a few minutes. The solution suggested is 800 grammes of saturated sodium sulphate +200 grammes of saturated sugar syrup. It is extremely difficult to accept this scientifically, as he quotes no statistics of clinical results and the scientific basis for his claims is incorrect. He states that a porcelain-like burn of the cornea occurs only in the horse and the rabbit because they have no lacrimal glands. This porcelain-like burn is recorded in the Medical History of the Great War in the 10 per cent. of severe cases in human beings, and the horse and the rabbit have lacrimal glands-the lacrimal bones are notched for the duct (E. A. Spaul, personal communication).

Bonnefon also suggests earlier in his paper that the tears remain caustic for a few days in any case. A hypertonic solution in the ordinary way would withdraw water only through the living semi-permeable membrane. It is possible, therefore, that his hypertonic solutions merely act in the same way as sodium bicarbonate-that is, as a mechanical irrigant. Nevertheless as he makes strong claims for the clinical results it would be interesting to analyse the washings of eyes burnt by D.E.S.- one group being irrigated with sodium bicarbonate and the other treated by hypertonic solutions. This might give us a clue as to the relative value of the two methods, since it is doubtful if the mere production of further oedema will do very much good. The induction of conjunctival oedema is, I think, disappearing as a treatment from the armoury of most ophthalmic surgeons these days.

\section{Summary}

The tolerance of the eye to certain reagents capable of neutralizing mustard gas under various conditions is discussed.

Animal research on the following lines is suggested: (1) The effect of the application of the reagents discussed above to eyes exposed to low concentrations of mustard gas. (2) The comparison of the washings of such eyes of the first few days for total chlorides, after treatment by $(a)$ alkaline lavage ; (b) hypertonic solutions, as suggested by Bonnefon.

The results thus obtained might indicate further clinical trial of some of these reagents in the event of gas warfare.

I am obliged for information from Professors Spaul and Challenger of Leeds University and Mr. B. Wood, senior dispenser of the General Infirmary at Leeds.

\section{REFERENCES}

Bonnefon, G. (1939). Gaz. hebd. Sci. méd., 60, 168.

Hederer, C., and Istin, M. (1935). L'Arme Chimique et ses Blessures, Baillière, Paris.

Kinttof, W. (1939). Experiments in Warfare Chemistry, London.

Kinttof, W. (1939). Experiments in Warfare Chemistry, London.
Mann, F. G., and Pope, W. J. (1922). J. chem. Soc., 121, 1053.

Official History of the War. Medical Services: Diseases of War (1923), 2, 49, London.

Peters, R. A., and Walker, E. (1923). Biochem. J., 17, 260.

Sollman, T. (1919). J. Pharmacol., 12, 303.

Sorsby, A. (1939). British Medical Journal, 2, 642

Thuillier, H. F. (1939). Gas in the Next War, London.

Warthin, A. S., et al. (1918). J. Lab. clin. Med., 4, 785.

\section{A RAPID PRESUMPTIVE SEROLOGICAL TEST FOR WEIL'S DISEASE}

BY

\section{H. C. BROWN, C.I.E., M.B., D.T.M. \& H.}

(From the Wellcome Bureau of Scientific Research, London)

The macroscopic agglutination of bacterial suspensions in the presence of homologous sera on a slide, which is gently rocked to and fro about a longitudinal axis, has been known for more than thirty years as a rough-andready method for the determination of the nature of a bacterium. Garrow (1917) elaborated the procedure in the form of his clockwork agglutinometer. It is claimed that the readings are on the average within one dilution of that found by the full macroscopic method, but, as the Medical Research Council's Report No. 51 points out, this represents a possible 100 per cent. error, and concludes that the field of usefulness of this apparatus lies where rapid work is required under stress of circumstances. It is exactly under such stress that the test described below is intended for use.

In the first place brief reference must be made to the standard agglutination technique devised by Professor Schüffner. This consists in making varying dilutions of the patient's serum, to which are added equal volumes of a formolized suspension of leptospira: after incubation, droplets are examined microscopically by dark-field illumination with a $2 / 3$-inch objective and a high eyepiece; no coverslip is used. I have personally for several years been in the habit of examining for agglutination after the mixture of leptospira and diluted serum has been allowed to interact overnight at room temperature ; other observers incubate for varying periods at different temperatures.

From time to time a macroscopic technique has been advocated similar to that used when studying bacterial agglutination, the results being read either by the naked eye or with a hand lens. Pot (1936), in describing a macroscopic test for Weil's disease, advocated the use of an antigen grown in Korthof's medium and incubated at $52^{\circ} \mathrm{C}$. for two hours, the results being read with a hand 
lens against a black background. In discussing this technique Brown and Broom (1939) gave it as their opinion that the test was not so sensitive as the microscopical test and that certain sera of low titres, such as those met with about the sixth day of the disease, might be overlooked when employing the macroscopic test. Brown and Broom also pointed out that human strains of Leptospira icterohaemorrhagiae isolated in England showed considerable differences in their agglutinability when using Schüffner's technique.

These observers also drew attention to the occasional occurrence of false positive reactions met with in the case of certain sera; these would cause difficulty in reading the macroscopic test. It is interesting to note that on the rare occasions upon which this false agglutination has been encountered a temporary false Wassermann reaction has also occurred. Even taking this and variations of agglutinability of 'different strains into account, the following rapid presumptive test would seem to be a useful diagnostic aid.

\section{Technique of the Test}

The test essentially consists in rocking to and fro on a slide for ten minutes small quantities of varying dilutions of the patient's serum in presence of a heavy suspension of L. icterohaemorrhagine; this saline suspension is formolized to a concentration of 0.2 per cent. The preparation of a suitably concentrated antigen for this test is not a simple matter, as leptospira cannot be easily thrown down by means of a centrifuge from the fluid medium in which it is grown. Centrifuging a culture of leptospira in Fletcher's broth at 5,800 revolutions per minute for half an hour does not cause any obvious clearing of the supernatant fluid.

There are numerous references in the literature to spinning down leptospira from fluid media, but in my experience it is not a practical proposition unless some reagent is added which will facilitate the sedimentation of leptospira from the medium when it is centrifuged. This can conveniently be done by adding to the fluid medium saponin up to a total concentration of 1 in 1,000 . The addition may be made after the culture has been formolized, which makes the procedure free from any risk of infection, although the strain, like the one employed, may have been subcultured at fortnightly intervals for five years and be no longer pathogenic.

Reference has already been made to the variation in agglutinability of various strains of leptospira which have been isolated from human cases during the last few years. Four human strains were compared as regards their agglutinability, both when put up in a water-bath for three hours at $56^{\circ} \mathrm{C}$. and on the rocking slide. The same serum was used in each case. Both procedures gave exactly the same titres, but the titres varied according to the strain used. One strain was agglutinated up to 1 in 1,000 , one only to 1 in 30 , and the two other strains up to 1 in 100 .

It is thus evident that the strain to be used as antigen in serological tests must be carefully chosen. The massed culture, about $500 \mathrm{c.cm}$., after the addition of formalin and saponin, is centrifuged at high speed and the deposit collected; this is suspended in saline until the opacity approximates that of a No. 1 Wellcome opacity tube used for the standardization of vaccines. The concentration required to get a suitable antigen from a well-grown culture for the rocking-slide method is approximately tenfold, so that $50 \mathrm{c.cm}$. of antigen can be obtained from half a litre of culture. This antigen is then centrifuged at approximately 3,000 revolutions per minute for ten minutes in order to get rid of any clumps of leptospira, and the supernatant fluid is bottled for future use.

The dilutions of the serum are made in the depressions of a painter's palette as in Schüffner's method. They are then mixed with an equal volume of antigen and placed on the slide shown in the diagram. This is made to rock for

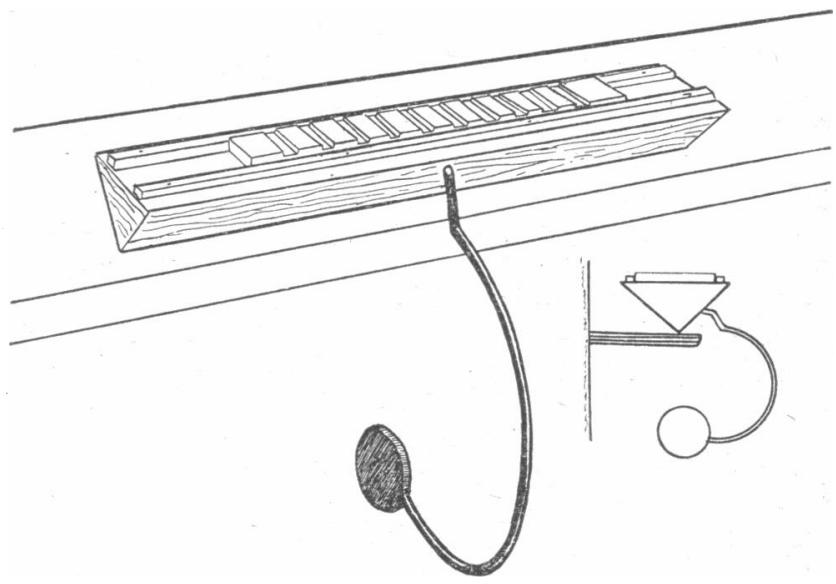

ten minutes, and the result is read by means of a hand lens against a dark background. Numerous sera have been tested by this method, and by the macroscopic method after incubation for three hours in a water-bath at $56^{\circ} \mathrm{C}$. The results have been compared with those obtained with Schüffner's technique, incubation having taken place at room temperature for approximately eighteen hours.

\section{Results}

The results of the rocking-slide method and the macroscopic have been consistently identical, but in every instance Schüffner's technique has given a titre three times higher than that obtained by either of the other methods. In order to test the sensitivity of the rocking-slide method two rabbits were immunized with $2 \mathrm{c.cm}$. of a broth culture of leptospira administered intravenously. Four days later the sera of these animals were tested by all three methods. The macroscopic and rocking-slide gave a titre of 1 in 10 , whereas Schüffner's method gave 1 in 30 . On the seventh day the two former readings were 1 in 100 and Schüffner's method 1 in 300 , and on the tenth day 1 in 1,000 and 1 in 3,000 respectively.

It is claimed that the rocking-slide method, given a suitable antigen, will produce a result within fifteen minutes of the receipt of the serum, and that it is reliable enough to justify the administration of curative serum.

I should like to thank my assistant, Mr. R. J. Reed, for his skill and care in maintaining the cultures of leptospira.

REFERENCES

Brown, H C., and Broom, J. C. (1939). British Medical Journal, 1,1178 .

Garrow, R. P, (1917). Lancet, 1, 262

Pot, A. W. (1936). Ibid., 1, 1290.

Histology of Mongolism.-Ten cases are described in which histological examination of the brain revealed widespread proliferation of the fibrous glia, which in several cases was not accompanied by corresponding changes in the myelin and in the cells. The pathogenesis of these changes is discussed, and it is suggested that the findings are not likely to represent the pathological substrate underlying mongolism. They do throw light, however, on the interpretation of certain previous findings.- Histological Changes in the Brain in Mongolism. A. Meyer and T. B. Jones.-J. ment. Sci., March, 1939, 85, 206. 